\title{
Pronunciación en lengua extranjera e identidad
}

\author{
LIDIA USÓ VICIEDO
}

UNIVERSITAT DE BARCELONA

ABSTRACT: This paper explores the association between pronunciation and identity in the context of teaching/learning a foreign language. Many foreign language students try to achieve a native accent so as to be accepted by others implies to the group of speakers from the target language; prefer to keep their own accent as a mark of identity. Thus, this work emphasizes how speaking a foreign language a change of identity, both socially and personally. It also illustrates some results of research about the beliefs that teachers of Spanish as a foreign language hold on the teaching/learning of pronunciation (Usó, 2007).

Keywords: pronunciation and identity, foreign accent, sociocultural identity, identity in a foreign language, foreign language learning.

RESUMEN: El presente trabajo explora la asociación existente entre pronunciación e identidad en el marco de la enseñanza/aprendizaje de una lengua extranjera. $\mathrm{Mu}-$ chos de los alumnos de lenguas extranjeras se decantan por intentar conseguir un acento nativo para pertenecer así al grupo de hablantes de esa lengua meta o bien, por el contrario, prefieren mantener el propio acento extranjero como señal de identidad. Así, en este trabajo se hace hincapié en cómo hablar una lengua extranjera supone un cambio de identidad, tanto a nivel social como personal. Se relaciona y ejemplifica con algunos resultados de una investigación acerca de las creencias de profesores de español como lengua extranjera sobre la enseñanza/aprendizaje de la pronunciación (Usó, 2007).

Palabras clave: pronunciación e identidad, acento extranjero, identidad sociocultural, identidad en lengua extranjera, aprendizaje de lenguas extranjeras. 


\section{Introducción}

En la historia de la enseñanza de lenguas extranjeras la importancia que se le ha dado a la pronunciación ha variado enormemente desde el Método de Gramática y Traducción, ${ }^{1}$ en el que no se la tenía en cuenta, hasta el Audiolingüismo, ${ }^{2}$ en el que pasó a tener un papel principal. El acento nativo era el objetivo en aquel momento.

Tras el abandono del método audiolingual, la pronunciación pasó por épocas de olvido (años 70 y 80 ). ${ }^{3}$ Se produjo un período de transición donde aparecieron tentativas que no gozaron de tanta aceptación o implantación, como la Sugestopedia, el Método de Curran, o Aprendizaje por Consejo, o el Método de Respuesta Física Total entre otras, que no prestaban demasiada o ninguna atención por la enseñanza de la pronunciación, ${ }^{4}$ aunque algunas de ellas se centrasen en una fonética correcta.

Finalmente, desde la década de los años 90, con el enfoque comunicativo, parecía que la pronunciación iba a recuperar relativamente su importancia al darse supremacía a la comunicación oral. ${ }^{5}$ Sin embargo, desde este momento, el principal objetivo pasó a ser la eficacia comunicativa, la fluidez y la inteligibilidad en la lengua extranjera. Por tanto, pasaban a un segundo plano el acento nativo e

1. El tradicional Método de Gramática y Traducción consistía en el estudio de reglas gramaticales, listas de vocabulario, traducción y comprensión de textos.

2. El Audiolingüismo (o método del ejército) surge durante la Segunda Guerra Mundial cuando el ejército americano necesitaba con urgencia hablantes de otras lenguas extranjeras. La lengua era considerada como hablada. Primero había que dominar las destrezas orales para luego pasar al dominio de las escritas. Los diálogos eran la forma preferida de presentar la lengua y se prestaba una gran atención a la pronunciación cuidada y lo más próxima a la nativa a través del contacto con informantes nativos y el laboratorio de idiomas. El conductismo de Skinner fue el soporte de este método en términos de aprendizaje. Se trataba de automatizar las estructuras básicas a través de ejercicios de repetición de menor a mayor dificultad.

3. Göbel y Graffman (1977) llamaron «hijastra» de la enseñanza de lenguas a la fonética, al poner de manifiesto la poca atención que recibía en esos años. Cabe señalar que se referían a la fonética y a la pronunciación como si se tratara de lo mismo, esto es, el estudio básicamente de los sonidos, aislados y en contacto.

4. En la actualidad hay que decir que la pronunciación se entiende como la producción y percepción tanto de los sonidos, como del acento, el ritmo y la entonación del habla (Kenworthy, 1987; Dalton y Seidlhofer, 1994; Seidlhofer, 2001; Cantero, 1998, 2003).

5. En estos años empiezan a aparecer algunos manuales sobre la pronunciación del inglés en los que se intenta enseñar pronunciación a través del enfoque comunicativo, aunque siguen apoyándose, en mayor o menor medida, en textos escritos y, en algunos casos, continúan utilizando el alfabeto fonético. Entre otros podemos citar Brown (1992), Taylor (1993), Dalton y Seidlhofer (1994) y Celce-Murcia et al. (1996). Sin embargo, en el caso concreto de la enseñanza-aprendizaje del español como lengua extranjera la influencia del desarrollo metodológico que operaba en Estados Unidos y en el resto de Europa fue demasiado tardía y llegó con poco empuje. 
incluso otros aspectos de la pronunciación. En consecuencia, actualmente se tolera el acento extranjero al no entorpecer normalmente la comunicación (Bartolí, 2005), o no haber una relación tan directa (Derwing y Munro, 2005).

Todo esto ha llevado a autores como Levis (2005) a afirmar que la historia de la pronunciación en lengua extranjera ha sido una historia «in extremes». ${ }^{6}$

En consecuencia, también la preocupación por la pronunciación, tanto de alumnos como de profesores de segundas lenguas y extranjeras, ha ido oscilando básicamente entre estos dos polos. Por una parte, encontramos la obsesión por conseguir un acento nativo y alcanzar la excelencia en pronunciación; ${ }^{7}$ por otra, la permisividad del acento extranjero, al no impedir generalmente la comunicación, provocada muchas veces por el escepticismo, tanto de unos como de otros, ante la ineficacia de la enseñanza de la pronunciación en el aula de lenguas extranjeras, así como de los métodos y manuales utilizados (Usó, 2007, 2009).

Sea como sea, lo cierto es que esta disyuntiva entre acento nativo y extranjero en la pronunciación de una lengua extranjera $(\mathrm{LE})^{8}$ está relacionada también con la idea de identidad, ya sea por querer adquirir un acento nativo para pertenecer al grupo de hablantes de esa lengua meta, como por no querer identificarse y preferir mantener el propio acento extranjero como rasgo identitario.

Seguidamente hablaremos de esta asociación entre pronunciación e identidad en LE y de la mencionada disyuntiva entre acento nativo o extranjero, relacionando y ejemplificando en ocasiones con muestras de discurso obtenidas en una investigación que realizamos, con profesores en formación de español como lengua extranjera (E/LE), ${ }^{9}$ acerca de sus creencias sobre la enseñanza/aprendizaje de la pronunciación (Usó, 2007).

\section{2. ¿Qué es el acento extranjero?}

El acento extranjero está considerado en la actualidad como un fenómeno natural, característico de la interlengua de los que aprenden una lengua extranjera.

Según Cantero (1994), el acento local o extranjero no se debe únicamente a una pronunciación particular o «incorrecta» de los sonidos, sino a una carac-

6. Para una revisión más exhaustiva de los distintos períodos por los que ha pasado la enseñanza de la pronunciación hasta la actualidad véase por ejemplo Celce-Murcia et al. (1996).

7. Véase Usó (2007) para una revisión de cómo entienden los profesores de E/LE el concepto de excelencia en pronunciación, de si se corresponde con el acento nativo, casi-nativo o con una idea máxima de corrección que no se identifica con el nativo.

8. A partir de ahora utilizaremos LE al referirnos a lengua extranjera.

9. A partir de este momento, emplearemos $\mathrm{E} / \mathrm{LE}$ al referirnos al español como lengua extranjera. 
terística integración entonativa de toda la materia fónica, se pronuncie o no correctamente.

Los sonidos se agrupan en bloques fónicos y no todos tienen la misma importancia. Por eso, a pesar de que los aprendices de la LE puedan pronunciar correctamente cada uno de los sonidos, conservan normalmente un acento extranjero, al organizar el discurso fónico según su propia lengua materna (Cantero, 2003). De hecho, no faltan estudios que defienden que la mayoría de la gente que adquiere una segunda lengua después de la infancia muestra unos patrones de pronunciación no nativos (Cantero, 2003; Derwing y Munro, 2005). El alcance y la naturaleza de tales acentos varía según la L1, la edad con la que se empezó a aprender la L2, la experiencia en la L2 y los factores motivacionales de cada alumno, entre otros (Piske, Mackay y Flege, 2001).

El acento extranjero no pasa desapercibido y puede condicionar la forma de actuar de los interlocutores nativos. En este sentido, Flege (1988) y Cortés (2002) señalan que el acento extranjero puede tener varias consecuencias para el hablante. Por un lado, puede ser una ventaja, ya que informa al interlocutor de que se encuentra ante un no nativo, y puede ser más tolerante y adaptar su producción oral para facilitarle la comprensión de su mensaje. ${ }^{10}$

Por otro lado, existen algunos inconvenientes como que el acento extranjero podría reducir la inteligibilidad del mensaje (Flege, 1988; Laroy, 1995). ${ }^{11}$ Además, una mala pronunciación exigirá una mayor atención por parte del interlocutor, y puede llevarle al cansancio o a la pérdida de paciencia. Por el contrario, existe la creencia generalizada de que una buena pronunciación supondrá una mayor aceptación por parte de los nativos (Cortés, 2002; Dieling y Hirschfeld, 2000), y se erigirá como tarjeta de presentación ante la sociedad, ya que una buena pronunciación suele ser digna de admiración y de elogio.

Por último, no podemos olvidar que existen ciertos estereotipos y prejuicios acerca del acento extranjero o «discurso acentuado» del no nativo (Derwing, Rossiter, Munro y Thomson, 2004). Derwing y Munro (2005) señalan que los hablantes nativos son muy sensibles a las producciones de los no nativos, detectan fácilmente variaciones segmentales, así como suprasegmentales. Sus reacciones ante un discurso marcado acentualmente son complejas y se pueden entender a muchos niveles. Así, en ocasiones, una mala pronunciación o un fuerte acento extranjero podría llevar a una evaluación social y una actitud negativa en los interlocutores e incluso, en algunos casos, a la burla y a la discriminación (Lippi-

10. Es lo que se ha llamado «Input modificado» (Gass y Varonis, 1984).

11. Aunque se asume normalmente que un acento muy marcado reduce automáticamente la inteligibilidad y la comprensibilidad, la relación no es tan directa según Derwing y Munro (2005). 
Green, 1997; Munro, 2003), al estar asociado a la identidad social y geográfica (Dalton y Seidlhofer, 1994; Laroy, 1995; Seidlhofer, 2001; Moyer, 2004; Dauer, 2005; Levis, 2005; Jenkins, 2005).

Seguidamente profundizaremos en esta relación entre pronunciación en LE e identidad.

\section{La identidad de nuestra pronunciación}

Según Dalton y Seildhofer (1994), con todo lo que hacemos y dejamos de hacer, decimos u omitimos, consciente o inconscientemente, establecemos nuestra identidad. No cabe duda de que una de las herramientas por excelencia que usamos para transmitir nuestra identidad es la lengua y, de manera especial, nuestra pronunciación (Dalton y Seidlhofer, 1994; Laroy, 1995; Seidlhofer, 2001; Moyer, 2004; Dauer, 2005; Levis, 2005; Jenkins, 2005; Verdía, 2010).

Por un lado, Morgan (1987) señaló que la forma de pronunciar de la gente está estrechamente relacionada con las percepciones de los otros sobre su identidad sociocultural. La identidad sociocultural es un constructo que define la relación entre el individuo y el amplio entorno social y cultural (Sifakis y Sougari, 2005). El individuo es interpretado a través de su asociación con instituciones tales como las familias, escuelas y lugares de trabajo, y «con los miembros de un grupo que comparten una historia común, una lengua común, y formas similares de entendimiento del mundo» (Norton, 1997: 420).

Según Moyer (2004), la pronunciación es un importante rasgo de identidad sociocultural en la medida en que es una característica lingüística adquirida en la infancia, solidificada en la temprana adolescencia y que, generalmente, está fuera del control cognitivo consciente o inmediato del adulto. Así, la forma de pronunciar de los alumnos de LE evidencia su identidad sociocultural (Laroy, 1995).

Además, nuestra forma de hablar transmite mucho de nosotros mismos: no es solo la expresión de nuestra identidad, sino que es parte de ella. De hecho, como menciona Verdía (2010), aspectos como el timbre, el tono, la entonación, la acentuación, el ritmo, la voz, etc., proporcionan a nuestros interlocutores mucha información sobre nosotros. Por una parte, les permite hacerse una idea de nuestra edad, sexo, origen, clase social y nivel de instrucción y, por otra, forjarse una opinión sobre nuestros rasgos de carácter y personalidad, como nuestra confianza, seguridad, timidez, ansiedad, simpatía, humor y estado anímico.

Por otro lado, al expresarnos en una lengua extranjera con un acento que nos parece próximo al de un nativo, la imagen que damos no siempre corresponde 
con nuestra autoimagen, ${ }^{12}$ es decir, con la visión que tenemos de nosotros mismos y que conforma nuestra identidad personal (Pukey y Novak, citado por Williams y Burden, 1999). Esto se debe a que a lo largo de nuestra vida nos vamos forjando una imagen de nosotros que no solo nos esforzamos por mantener ante los demás, sino que tratamos de reforzar y de proteger. ${ }^{13}$

Desde este punto de vista, podríamos sentir amenazada nuestra autoimagen si la imagen que proyectamos al hablar la LE no coincide con la que queremos dar a nuestros interlocutores. Además, este desequilibrio existente entre la propia proyección y la que perciben los demás puede ocasionar inseguridad y extrañeza.

En cierta manera, hablar una LE con la pronunciación de un nativo puede suponer para algunas personas renegar de su identidad o, al menos, ponerla en peligro (Verdía, 2010). En concreto, cuando los alumnos perciben una amenaza para su autoimagen es probable que se produzca una resistencia al aprendizaje, ya sea de manera consciente o inconsciente (Williams y Burden, 1999). No debemos olvidar que los lazos afectivos con nuestra lengua materna son normalmente positivos y fuertes (Laroy, 1995). Así, algunos alumnos pueden optar por mantener deliberadamente su acento distintivo para no perder su identidad, para ganar la aprobación de sus iguales o para no resultar pedantes frente al grupo, mostrando poco o ningún interés por acercarse a una pronunciación más próxima a la del hablante nativo (Verdía, 2010).

En estas ocasiones, Laroy (1995) mantiene que los profesores deberían respetar que los alumnos prefiriesen un acento no nativo.

En esta línea, Jenkins (2005) también hace referencia a profesores no nativos de ESL ${ }^{14}$ que se decantan por usar su acento extranjero en sus clases para expresar su identidad, su L1, o su pertenencia a una comunidad internacional de habla inglesa. ${ }^{15}$

12. La autoimagen es una de las tres dimensiones del autoconcepto, esto es, el sistema de creencias del individuo respecto a su existencia personal, desarrollado y aprendido a lo largo de su vida, que él cree verdadero y da consistencia a su personalidad (Pukey y Novak, citado por Williams y Burden, 1999).

13. Ya Goffman en 1967 se refirió a la noción de imagen para explicar el modo en el que nos relacionamos socialmente en los intercambios comunicativos. Para él la imagen no es lo que realmente somos, sino más bien la representación que creamos de nosotros mismos y que aspiramos a mostrar a los demás. En torno a este concepto de imagen se desarrollan toda una serie de rituales conversacionales para tratar de protegerla y de no dañar, al mismo tiempo, la imagen de nuestros interlocutores; o bien, de repararla o compensarla, si ya la hemos dañado. Esta noción de imagen ha sido una pieza clave para la propuesta teórica sobre la cortesía verbal de Brown y Levinson (1987).

14. English as a Second Language.

15. La globalización ha promovido el dominio del inglés en áreas internacionales tan importantes 
A este respecto, Levis (2005) alude al acento como un marcador esencial de pertenencia social. Así, en su opinión, los hablantes hablan según el grupo social al que pertenecen o desean pertenecer.

En la investigación que realizamos con profesores de E/LE en formación (Usó, 2007) algunos de ellos se manifestaron a favor de querer mantener su identidad mediante su acento extranjero a la hora de hablar una LE, esto es, cuando actúan como hablantes, y algunos como los alumnos que todavía son, de LE:

Yo prefiero hablar inglés como si hablara español, porque no me identifico con la otra lengua y prefiero mantenerme en mi sitio. Cuando hablo la LE, ¿soy el mismo? El timbre de mi voz, ¿cómo suena? ¿Qué imagen daré? Puede haber estudiantes que piensen así. (Inf.A1)

Además, algún profesor de los estudiados pertenecía a una variedad no prestigiosa, ${ }^{16} \mathrm{o}$ alejada del estándar oral del español, y rehusaba adoptar dicha variedad estándar a la hora de dar sus clases. De esta manera, quería mantener también su propio acento, el de su variedad, no queriendo renunciar a su identidad:

Un profesor debe ser lo que es y enseñar la lengua de la manera más natural, es decir, «la propia». Soy de Buenos Aires. (Inf.A5)

Si tenemos en cuenta que las recientes teorías cognitivas perciben la enseñanza/aprendizaje como un proceso activo y constructivo que está influido por las creencias de los individuos, se podría intentar seguir en esta línea de investigación, tanto con profesores como con alumnos, para averiguar su posicionamiento acerca de querer mantener el acento extranjero por ser una muestra de identidad, en la medida en que ello pueda condicionar tanto la manera de enseñar la pronunciación de los docentes, como la adquisición/aprendizaje por parte de los aprendices.

Sin duda, esta preferencia por querer mantener el propio acento extranjero y expresar así la propia identidad nos llevaría al tema de las actitudes lingüísticas de los hablantes hacia ciertas variedades, acentos o lenguas. Pero no nos centra-

como los medios de comunicación, la educación, las relaciones internacionales, los viajes y la seguridad (Crystal, 2003). Está cada vez más extendido el uso del English as an Internacional Language (EIL). Este término se refiere a las variedades del inglés, estandarizadas o no, que se usan para la comunicación entre hablantes no nativos de inglés (Sifakis y Sougari, 2005). Se basa en la necesidad de crear un discurso que sea comprensible para los interlocutores a diferentes niveles de competencia lingüística y en toda una amplia gama de situaciones comunicativas (Jenkins, 2004; Seidlhofer, 2004; Sifakis, 2004).

16. Aludimos aquí al concepto de variedad no prestigiosa únicamente en oposición a la norma estándar y al prestigio que se asocia a esta normalmente (Fernández Moreno, 1997; Hernández Alonso, 2001; Carbó et al., 2003). 
remos ahora en ello, aunque es una vía de estudio que merece también ser tratada en profundidad en el campo de las lenguas extranjeras. ${ }^{17}$

Ahora bien, respecto a la idea de no querer perder la identidad queremos resaltar cómo para alguno de los profesores de nuestro estudio la defensa del acento extranjero y la inteligibilidad del mensaje eran, en realidad, una manera de consolarse ellos mismos como hablantes/alumnos de LE al no poder llegar a conseguir la excelencia en pronunciación, o no saber cómo hacerlo.

Mostramos aquí un ejemplo de esta contradicción entre creencias de las que hablamos y que reveló alguno de nuestros informantes:

[...] no se persigue la excelencia, sino la inteligibilidad comunicativa $\neq$ Sufro en carne propia la realidad de no poder realizar determinados sonidos. Me consuelo diciendo que lo importante es entender y hacerse entender. (Inf.A5)

Esto evidenciaría la existencia de contradicciones e incongruencias en el sistema de creencias del profesor. De hecho, muchas de las creencias que manifestamos, que creemos que rigen nuestra conducta en el aula y que podemos explicitar en un momento dado, pueden verse contradichas por otras menos conscientes o implícitas, o bien por nuestra práctica educativa (Nunan, 1987; Fang, 1996; Basturkmen et al., 2004; Usó, 2007). Creemos que sería interesante seguir profundizando también en esta vía de estudio.

\section{Pronunciar en LE. ¿Un cambio de identidad?}

Otra cuestión que nos interesa destacar es el hecho de que, por el carácter social de la lengua, aprender a hablar otro idioma es diferente del aprendizaje de otras materias. Aprender un idioma no es solo aprender a usar una nueva gramática, aplicar unas reglas sintácticas y memorizar piezas léxicas, supone también adoptar nuevas conductas sociales y culturales características de otra comunidad (Guiora, citado por Celce-Murcia et al., 1996). Tenemos que ser conscientes de que es un cambio en la naturaleza social del individuo. En este sentido, el alumno tiene que modificar su forma de ser y acostumbrarse a verse de otra forma, sentirse diferente, reconocerse en su nueva imagen y aceptarse a sí mismo. Al aprender a hablar una lengua extranjera el alumno tendrá, por lo tanto, que construir un nuevo yo social. Desde este punto de vista, Verdía (2010) sostiene que expresarse en una nueva lengua supone un cambio de identidad.

En este sentido, ya Goffman (1967) entendió la actividad comunicativa en la propia lengua como una especie de obra teatral en la que los participantes in-

17. Para una amplia revisión sobre el tema de actitudes lingüísticas véase Blas (1999). 
terpretan un papel que se va definiendo a medida que avanza la interacción. Así pues, resulta comprensible y coherente pensar que «aprender una lengua que no es la nuestra es en el fondo aprender a ser una persona social distinta», (Crooball y Oxford, citado por Williams y Burden, 1997: 123).

Otro aspecto importante relacionado con ese cambio de identidad y que debemos tener en cuenta es que la producción de sonidos conlleva sobre todo aspectos físicos y está íntimamente ligada a nuestro cuerpo. Cuando hablamos no solo entran en juego la cara, la lengua, la laringe, el tórax, etc. (Lleó, 1997), que intervienen en la producción de sonidos, sino que también hay otros aspectos físicos que intervienen para acompañar nuestra producción, como movimientos oculares, expresiones faciales, movimientos de cabeza, gestos, etc., ya que usamos el cuerpo completo para comunicarnos con los demás.

Así, según Verdía (2010), al hablar una lengua extranjera también nos sentimos físicamente diferentes, incluso podemos llegar a sentirnos ridículos al producir esos «extraños sonidos» para los que tenemos que realizar diferentes muecas, redondear o estirar los labios, mostrar la lengua o producir sonidos velares, guturales, nasales, etc. a los que no estamos acostumbrados. Hacer todo esto cuando pronunciamos una LE se asemeja bastante a la interpretación de una obra teatral. Además, puede producir inhibición, vergüenza y miedo en el alumno y limitarle en su adquisición/aprendizaje de la pronunciación. En otras palabras, hablar una lengua extranjera activa toda una serie de factores emocionales que sin duda no ayuda a cambiar de identidad.

Aun así, hay gente que, por su personalidad, posee mayor capacidad de adaptación para renunciar parcial o temporalmente a su individualidad, lo que le facilita identificarse con otros y adoptar identidades transitorias. Se habla de que estas personas tienen unas barreras del ego más débiles o de permeabilidad del ego (Guiora, citado por Liceras, 1992; Ehrmann, 2000), ${ }^{18}$ revelando también un mayor grado de aculturación (Shumann, 1976). ${ }^{19}$

En nuestro estudio (Usó, 2007) algunos profesores revelaron la creencia de que para ellos hablar una lengua extranjera es similar a una puesta en escena, a una representación teatral, como si se tratara de representar otro papel, una segunda identidad. Aludieron a toda esa serie de gestos, entonación, caras, etc. que conlleva, además de algunos componentes emocionales y barreras psicológicas, como miedos y vergüenzas.

18. Además, estas personas pueden llegar a adquirir una mejor competencia en la LE, según Guiora, citado por Liceras (1992).

19. El grado de aculturación es la capacidad que tiene el aprendiz de adaptarse a la cultura de la lengua objeto y, según ciertos estudios, parece jugar un papel importante en la adquisición de dicha lengua (Schumann, 1976). 
Según nuestros informantes, cuando se pasa a un estadio de apropiación de la lengua extranjera ya no se siente como ajena a uno, dejamos de sentirnos extraños cuando hablamos y pasamos a actuar como un nativo. Reproducimos aquí algunas muestras de sus discursos en este sentido:

Hablar un idioma que no es el tuyo es una puesta en escena, casi teatral, que conlleva toda una serie de componentes emocionales, y más cuando tienes espectadores de tu propia lengua. Por eso no es raro oír que con un par de cervezas hablo con más fluidez. Cuando se pasa al estadio de apropiación de la LE ya no se siente como ajena a uno, se puede bromear, opinar, etc., como en nuestra lengua materna. La competencia comunicativa alcanzada permite no sentirse extraño cuando se habla. (Inf.A3)

El miedo a no ser nosotros mismos cuando hablamos una LE se superaría fácilmente si en las clases fuera posible que todos los alumnos hablaran más. Al final cuando se convierte en hábito, uno se acaba sintiendo bien en esa segunda piel. (Inf.A7)

Os podría contar, con todo lujo de detalles, de todos esos complejos y extrañamientos de la propia personalidad. Estuve un año entero sin hablar más que lo puramente imprescindible. (Inf.A6)

Algunos de los profesores estudiados señalaron que una de las labores del docente es hacer que el alumno supere estas barreras psicológicas que hacen difícil hablar una LE, fomentando una imagen positiva del alumno, su autoestima, para que este se sienta seguro y pueda actuar en la LE como lo hace un nativo. Así, el profesor debería crear situaciones de habla donde el alumno no se sienta observado ni juzgado, ya que es muy importante para llevar a cabo un proceso de producción oral. De todas formas, pensamos que, si hubiera herramientas pedagógicas y una técnica efectiva y bien dirigida para enseñar la pronunciación, tal vez no sería tanta la influencia de los factores emocionales del alumno, ni tan delicada y casi psicoterapéutica la labor del profesor.

Otros profesores estudiados revelaron también contradicciones en su sistema de creencias acerca de ese cambio de identidad que supondría hablar y pronunciar en una LE. Conscientes como docentes de lo que conlleva actuar en una nueva lengua, se mostraban reacios sin embargo a cambiar y/o perder su identidad cuando se referían a ellos mismos como hablantes de LE y a su pronunciación.

Vemos aquí las contradicciones de dos de los informantes, uno de ellos citado anteriormente (ver apartado 3), donde se ve claramente cuáles son sus creencias como profesores y lo que ellos creen y hacen como hablantes/alumnos de LE: ${ }^{20}$

20. Para más información sobre las posibles contradicciones en el sistema de creencias de los profesores de LE y, en concreto, entre la visión del profesor y la de este como hablante/alumno de LE véase Usó (2007). 
Pronunciar correctamente conlleva una serie de gestos, entonación, caras [...] que puede parecer ridículo a algunos alumnos, implica actuar como lo hacen los nativos, siendo una persona nueva. $\neq$ Yo prefiero hablar inglés como si hablara español, porque no me identifico con la otra lengua y prefiero mantenerme en mi sitio. Cuando hablo la LE ¿soy el mismo?, el timbre de mi voz, ¿cómo suena? ¿Qué imagen daré? Puede haber estudiantes que piensen así. (Inf.A1)

Hablar una LE es, en cierto modo, similar a una representación teatral y trae, por tanto, muchos miedos y vergüenza. $\neq$ Reconozco que no me preocupa mi acento extranjero, es más, me gusta, ya que si no fuese así estaría fingiendo algo que no soy. (Inf.A11)

También respecto a este tema de que hablar una LE sea representar otro papel, pensamos que haría falta más investigación. La idea de que se está actuando cuando se habla una lengua extranjera y de que construimos una segunda identidad, donde hay que entonar, pronunciar y gesticular de otra manera a como lo hacemos en nuestra lengua materna, es un tema en el que se debería profundizar más. $^{21}$

\section{La imitación a los nativos como camino para cambiar la identidad en LE}

Por último, otro aspecto que nos gustaría comentar aquí es la idea de la imitación a los nativos, que apuntaron los profesores en nuestra investigación (Usó, 2007), como una buena manera para adquirir la pronunciación de la LE (entonación, acento, ritmo, sonidos, etc.). De este modo, se podría ir venciendo esa resistencia a perder la propia identidad de la que hablábamos anteriormente y adaptándose a la nueva. Reproducimos aquí algunas de las creencias que manifestaron a este respecto:

Hay que animar a nuestros estudiantes para que hablen español imitando a los españoles. Así, casi sin darse cuenta, y venciendo la resistencia a perder la propia identidad, el estudiante iría adquiriendo muchos de los sonidos y entonación de la lengua meta. (Inf.A1)

Hay un gran componente imitativo en el proceso de aprendizaje de la producción oral, e incluso de oído musical. Por ejemplo, un inglés que aprende español en Granada, seguramente no pronunciará todas y cada una de las letras que componen

21. En este sentido nos gustaría mencionar a Orta (2009 b) quien sugiere recurrir a algunas técnicas teatrales para enseñar la pronunciación de la LE en clase, y poner así en relación la puesta en escena con la actuación en la LE. 
una palabra, o mejor, las pronunciará pero con variantes de pronunciación como hacemos también en Murcia. (Inf.A3)

Mi aprendizaje de la pronunciación ha sido escuchando e imitando a los hispanohablantes, no por las horas escuchando la lengua en el laboratorio de fonética. (Inf.B6).

En relación con este tema, Carbó et al. (2003) mencionan que existe la falsa creencia de que si el alumno aprende la lengua en el país, puede aprender a pronunciar por emulación, pues tiene, además de su profesor como modelo, el contacto directo con hablantes nativos. ${ }^{22}$ De hecho, no faltan voces que afirman que no se puede adquirir una buena pronunciación de la LE sin pasar por una inmersión o exposición a la lengua meta.

Impera también la creencia entre una gran mayoría de docentes de que lo que realmente importa es que el alumno tenga una buena base gramatical y léxica y que ya desarrollará la lengua oral, y en concreto la pronunciación, cuando decida pasar una temporada en el país de la lengua meta.

En nuestro estudio muchos de los profesores defendieron que la excelencia en pronunciación pasa por la inmersión en el país de la LE. Veamos algunos ejemplos a este respecto:

Desconozco métodos que hagan posible una pronunciación cuidada sin antes pasar por una inmersión temporal en la cultura de la lengua meta. La entonación es muy difícil de enseñar y aprender. Es el resultado de la exposición variada y continua a la lengua meta. (Inf.C1)

No podemos pretender que nuestros alumnos alcancen la excelencia desde el primer momento, ya que es muy difícil, casi imposible de alcanzar una vez llegada cierta edad, sin vivir en el país donde se habla la L2, sin estar en contacto frecuente con hablantes de la L2. Además poco podemos hacer si el alumno se limita al tiempo de clase. Su pronunciación mejorará y se acercará a la excelencia si se esfuerzan por escuchar canciones y textos en español, por ver películas, relacionarse con hispanohablantes, vivir en el país. (Inf.B7)

22. Tal vez por ello estos autores creen que existe el contraste entre manuales de E/LE publicados en España y aquellos publicados en el extranjero respecto al tratamiento de la pronunciación. En los primeros la atención al tema es escasa, a diferencia de los segundos, donde ocupa una parte considerable (Amann et al., 1994; Blanco et al., 2001). Estos últimos suelen basarse en el análisis contrastivo entre el sistema fonológico de esta lengua y la del estudiante. Además los fenómenos suprasegmentales se abordan ya desde las primeras lecciones. 
Pretender conseguir la excelencia en la pronunciación con alumnos que no están inmersos en la cultura es completamente ilusorio. (Inf.C5)

Mi experiencia me dice que en el proceso de adquisición de la pronunciación es más difícil enseñar aspectos como la entonación, ritmo, acento que la correcta pronunciación de un fonema. Para ello sí que ayuda un proceso de inmersión en la LE. (Inf.C13)

[...] lo que me ha ayudado a mejorar mi pronunciación no fue sino el oír, una y otra vez, a hablantes nativos con distintos acentos en situaciones reales y mantener comunicaciones con ellos. (Inf.A11)

Estas creencias de los profesores, fundamentadas muchas veces sobre sus experiencias como hablantes/alumnos de LE, o provocadas tal vez por los malos resultados obtenidos en el aula y por la propia desconfianza en los métodos y manuales existentes, ${ }^{23}$ condicionan inevitablemente la didáctica de la pronunciación, relegándola a un plano secundario (Bartolí, 2005). En consecuencia, tal y como señalan Kenworthy (1987) y Moyer (2004), el papel del profesor en la enseñanza de la pronunciación se suele ver como algo relativamente poco importante, al percibirse además la pronunciación como un rasgo notable de la personalidad lingüística del individuo que no se puede controlar conscientemente. Tan solo factores como la exposición a la lengua meta y el contacto con nativos, la actitud o la motivación, se suelen considerar factores potenciales en la mejora de la pronunciación.

En nuestra opinión, creemos que es necesario averiguar las creencias de los profesores y de los alumnos respecto a cómo y dónde mejor aprender la pronunciación, en la medida en que estas condicionan el propio proceso de enseñanza/ aprendizaje, para así intentarlas modificar, si conviene. Consideramos esto como un paso previo y necesario para lograr mejores resultados en este sentido.

Así también, Derwing y Munro (2005) aluden a la falta de estudios desde el campo de la lingüística aplicada que evalúen la eficacia de la enseñanza comunicativa de la pronunciación en el aula y la posibilidad de la eliminación del acento extranjero. Además, en relación al acento nativo, o casi nativo, y a la posibilidad de poder alcanzarlo, o no, en la clase de LE, estos autores advierten que los profesores muestran una gran confusión sobre lo que es posible y deseable en la enseñanza de la pronunciación, poniendo en duda que este objetivo sea alcanzable

23. Los manuales existentes apenas prestan atención a la enseñanza de la pronunciación y, cuando lo hacen, no desligan pronunciación de corrección fonética y se basan en el conocido «escucha y repite», es decir, en la audición y la imitación. Además, todavía no ha habido propuestas de integración de la pronunciación en el enfoque comunicativo ni en el enfoque por tareas (Llisterri, 2003; Carbó et al., 2003; Derwing y Munro, 2005). 
en dicho contexto. En consecuencia, alegan que los alumnos que se empeñan por alcanzar un acento nativo se llegan generalmente a desanimar. ${ }^{24}$

Dauer (2005) también alude al desaliento de algunos profesores ante la incapacidad de sus alumnos para mejorar su pronunciación.

Con todo, es una realidad que la pronunciación sigue estando en gran medida desatendida tanto en la enseñanza comunicativa de las lenguas extranjeras como en la investigación (Cantero, 1994; Llisterri, 2003; Iruela, 2004; Derwing y Munro, 2005; Levis, 2005; Usó, 2007, 2009; Orta, 2009 a), además de estar supeditada todavía al conocimiento lecto-escritor (Cantero, 1994; Usó, 2007; Bartolí, 2005, 2012; Giralt, 2006, 2012). Pero hay quien cree que en el aula de LE se puede incluso acelerar el proceso de adquisición fónica, siempre que la enseñanza de la pronunciación esté bien dirigida (Bartolí, 2005, 2012; Giralt, 2006, 2012). En concreto, esta última autora aporta resultados positivos sobre la enseñanza/aprendizaje y adquisición de la pronunciación de E/LE en el aula, a partir de una investigación llevada a cabo mediante un enfoque oral, sin la mediación lecto-escritora y siguiendo el enfoque por tareas.

\section{Consideraciones finales}

Tras estas reflexiones, nos gustaría señalar la necesidad de profundizar en las creencias, tanto explícitas como implícitas, de nuestros alumnos de LE sobre la relación pronunciación e identidad. Nos interesa saber cómo se perciben ellos mismos hablando en la LE, sus posibles reticencias (razones identitarias) e inconvenientes (factores emocionales y barreras psicológicas) a la hora de interpretar y/o adoptar esa segunda identidad. Finalmente, creemos relevante averiguar también si su preferencia por el acento extranjero responde a un posicionamiento real y es fruto de una actitud lingüística, o, en realidad, estaría ocultando su frustración ante la imposibilidad de alcanzar el acento nativo y/o la falta de metodología y técnicas efectivas para lograrlo. En este sentido, resultaría interesante descubrir las posibles contradicciones existentes en el sistema de creencias de los alumnos. Todo ello nos ayudaría a la hora de dirigir la enseñanza de la pronunciación, en la medida en que sus creencias puedan actuar como un fuerte filtro respecto a su aprendizaje/adquisición.

Igualmente, es muy probable que los profesores actúen en base a creencias y prejuicios asentados en sus propias experiencias educativas como hablantes/ alumnos de LE y a las formadas en el contexto de enseñanza en el que desarro-

24. En este sentido, Jenkins $(2000,2002)$ afirma que el acento nativo es un objetivo poco realista e innecesario para la mayoría de los que aprenden una LE. 
llan su labor docente (Pajares, 1993; Golombeck, 1998; Cambra, 2000). Hacer emerger y acceder a las creencias, tanto de unos como de otros, es un buen punto de partida para empezar a dirigir y modificar tanto la actuación didáctica del profesor, en los cursos de formación, como el comportamiento psicolingüístico de los alumnos en el aula, respecto a la enseñanza/aprendizaje de la pronunciación (Usó, 2007, 2009).

\section{Referencias bibliográficas}

Amann, K. A.; S. Marín; E. Osorio (1994): Encuentros, Berlín, Cornelsen.

BARTOLí, M. (2005): «La pronunciación en la clase de lenguas extranjeras», Phonica, 1: 1-27. (2012): La pronunciación por tareas en la clase de E/LE, tesis doctoral inédita dirigida por Dr. Francisco José Cantero Serena, Universitat de Barcelona.

Basturkmen, H.; S. Loewen; R. Eldis (2004): «Teachers' Stated Beliefs about Incidental Focus on Form and their Classroom», Applied Linguistics, 25(2): 243-272.

Blanco, J. A.; M. A. Dellinger; P. Donley; M. I. García (2001): Vistas. Introducción a la lengua española, Boston, Vista Higher Education.

Blas Arroyo, J. L. (1999): «Las actitudes hacia la variación intradialectal en la sociolingüística hispánica», Estudios filológicos, 34: 47-72.

Brown, G. (1992): Approaches to Pronunciation, Teaching Modern English Publications in association with the British Council.

Brown, P.; S. C. Levinson (1987): Politeness. Some universals in Language Use, $2^{\mathrm{a}}$ ed., Cambridge, cup.

Cambra, M. (2000): «El pensament del professor: formació per a la pràctica reflexiva» en A. CAMP.; I. Ríos; M. CAMBRA (eds.) (2000): Recerca i formació en didàctica de la llengua, Barcelona, Graó.

CANTERo, F. J. (1994): «La cuestión del acento en la enseñanza de lenguas» en SÁnchez, J.; I. SANTos (eds.) (1994): Problemas y métodos en la enseñanza del español como lengua extranjera, Madrid, SGEL. (1998): «Conceptos clave en lengua oral» en Mendoza, A. (coord.) (1998): Conceptos clave en didáctica de la lengua y la literatura, Barcelona, Horsori. (2003): «Fonética y didáctica de la pronunciación» en MendozA, A. (coord.) (2003): Didáctica de la Lengua y la Literatura, Prentice Hall.

Carbó, C.; J. Llisterri; M. J. Machuca; C. de la Mota; M. Riera; A. Ríos (2003): «Estándar oral y enseñanza de la pronunciación del español como primera lengua y como lengua extranjera», ELUA, Estudios de Lingüistica de la Universidad de Alicante, 17: 161-180. $<$ http://liceu.uab.es/publicacions/carbo_et_al_EULA03.pdf $>$ 
Celce-Murcia, M.; D. Brinton; J. Goodwin (1996): Teaching Pronunciation: A Reference for Teachers of English to Speakers of Other Languages, New York, Cambridge University Press.

CoRTÉs, M. (2002): Didáctica de la prosodia del español: la acentuación y la entonación, Madrid, Edinumen.

Crystal, D. (2003): English as a Global Language, Cambridge, Cambridge University Press.

Dalton, C.; B. Seidlhofer (1994): Pronunciation, Oxford, Oxford University Press.

DAUER, R. M. (2005): «The Lingua Franca Core: A New Model for Pronunciation Instruction?», TESOL Quarterly, 39 (3): 543-550.

Derwing, T. M.; M. Munro (2005): «Second Language Accent and Pronunciation Teaching: A Research-Based Approach», TESOL Quarterly, 39 (3): 379-397.

Derwing, T. M.; M. J. Rossiter; M. J. Munro; R. I. Thomson (2004): «Second Language Fluency: Judgments on Different Tasks», Language Learning, 54: 655-679.

Dieling, H.; U. Hirschfeld (2000): Phonetik lehren und lernen, Langenscheidt.

Ehrmann, M. (2000): «Los límites del ego y la tolerancia a la ambigüedad en el aprendizaje de segundas lenguas» en ARNOLD, J. (ed.) (2000): La dimensión afectiva en el aprendizaje de idiomas, Madrid, CUP.

FANG, Z. (1996): «A Review of Research on Teacher Beliefs and Practices», Educational Research, 38 (1): 47-65.

Fernández Moreno, F. (1997): «¿Qué español hay que enseñar? Modelos lingüísticos en la enseñanza de español/LE», Cuadernos Cervantes de la Lengua Española, 14: 7-15.

Flege, J. E. (1988): "The Production and Perception of Foreign Language Speech Sounds» en WiniTz, H. (ed.) (1988): Human Communication and its Disorders, a Review, Norwood, Ablex. 224-401.

Gass, S.: E. M. VARonis (1984): "The Effect of Familiarity on the Comprehensibility of Nonnative Speech», Language Learning, 34: 65-89.

GiraLT, M. (2006): «El enfoque oral en la enseñanza del español como lengua extranjera: experiencia piloto de una propuesta didáctica», Phonica, 2: 1-26. (2012): El enfoque oral en la iniciación de la enseñanza/aprendizaje y adquisición de la pronunciación del español como lengua extranjera, tesis doctoral inédita dirigida por Dr. Francisco José Cantero Serena. DL: B. 31768-2012.

$<$ http://hdl.handle.net/10803/94136>.

Göbel, H.; H. GrafFMan (1977): «Ein Stiefkind des Unterrichts DaF: Ausprachschulung», Zielprache Deutsch, 3. 
Goffman, E. (1967): Interactional Ritual: Essays on Face Behaviour, New York, Garden City.

Golombeck, R. P. (1998): «A Study of Language Teachers' Personal Practical Knowledge», Teachers of English to Speakers of Other Languages Quarterly, 32 (3): 447-464.

Hernández Alonso, C. (2001): «¿Qué norma enseñar?» en Actas electrónicas del II Congreso Internacional de la Lengua Española, "El español en la Sociedad de la Información», Valladolid, 16-19 de octubre.

$<$ http://cvc.cervantes.es/obref/congresos/valladolid/ponencias/unidad_ diversidad_del_español/1_la norma_hispanica/hernandez_c.htm $>$.

Iruela, A. (2004): Adquisición y enseñanza de la pronunciación en lenguas extranjeras, tesis doctoral inédita dirigida por Francisco José Cantero Serena, Barcelona, Universitat de Barcelona.

Jenkins, J. (2000): The Phonology of English as an International Language, Oxford, Oxford University Press. (2002): «A Sociolinguistically-Based, Empirically-Researched Pronunciation Syllabus for English as an International Language», Applied Linguistics, 23: 83-103.

(2004): «Research in Teaching Pronunciation and Intonation», Annual Review of Applied Linguistics, 24: 109-125. (2005): «Implementing and International Approach to English Pronunciation: The Role of Teacher Attitudes and Identity», TESOL Quarterly, 39 (3): 535-543.

Kenworthy, J. (1987): Teaching English Pronunciation. Harlow, Longman.

LARoy, C. (1995): Pronunciation (Resource Books for Teachers), Oxford, Oxford University Press.

LeVIS, J. M. (2005): «Changing Contexts and Shifting Paradigms in Pronunciation Teaching», TESOL Quarterly, 39 (3): 369-377.

LiCERAS, J. M. (1992): La adquisición de lenguas extranjeras: hacia un modelo de la interlingua, Madrid, Visor (Lingüística y Conocimiento).

LIPPI-GreEN, R. (1997): English with an Accent: Language Ideology and Discrimination in the United States, New York, Routledge.

LLEó, C. (1997): La adquisición de la fonología de la primera lengua y de las lenguas extranjeras, Madrid, Visor.

LlisterRI, J. (2003): «La evaluación de la pronunciación en la enseñanza del español como segunda lengua» en ReyzábaL, M. V. (dir.) (2003): Perspectivas teóricas y metodológicas: Lengua de acogida, educación intercultural y contextos inclusivos, Madrid, Dirección General de Promoción Educativa, Consejería de Educación, Comunidad de Madrid. 547-562. $<$ http://liceu.uab.es/ joaquim/publicacions/Eval_Pron_EL2.pdf $>$.

Morgan, B. (1987): «Identity and Intonation: Linking Dynamic Processes in an ESL Classroom», TESOL Quarterly, 31: 431-450. 
Moyer, A. (2004): Age, Accent and Experience in Second Language Acquisition: An Integrated Approach to Critical Period Inquiry, Clevedon, Multilingual Matters.

Munro, M. J. (2003): «A Primer on Accent Discrimination in the Canadian Context», TESL Canada Journal, 20 (2): 38-51.

NorTON, B. (1997): «Language, Identity, and the Ownership of English», TESOL Quarterly, 31: 409-429.

NunAN, D. (1987): «Communicative Language Teaching: Making it Work», English Language Teaching Journal, 41: 136-145.

OrTA, A. (2009 a): La enseñanza explícita de la pronunciación. Creencias de los profesores y sus repercusiones en el aula de E/LE, Memoria de investigación, asesor: Raúl Alfonso, Universitat de Barcelona.

$<$ http://diposit.ub.edu/dspace/handle/2445/9823>.

(2009 b): Técnicas teatrales aplicadas a la enseñanza de la pronunciación, conferencia presentada en el VI Encuentro Práctico de Profesores de Español, International House, Wüzburg, Alemania.

$<$ http://www.youtube.com/watch?v=77xspAfZ31Y $>$.

Pajares, M. F. (1993): «Preservice Teacher's Beliefs: A Focus for Teacher Education», Action in Teacher Education, 15 (2): 45-54.

Piske, T.; I. R. A. MAcKay; J. E. Flege (2001): «Factors Affecting Degree of Foreign Accent in an L2: A Review», Journal of Phonetics, 29: 191-215.

SChumanN, J. (1976): «La adquisición de lenguas segundas: la hipótesis de la pidginización» en LiCERAS, J. M. (ed.) (1992): La adquisición de lenguas extranjeras: hacia un modelo de la interlingua, Madrid, Visor (Lingüística y Conocimiento).

Seidlhofer, B. (2001): «Pronunciation» en Carter, R.; D. Nunan. (eds.): Teaching English to Speakers of Other Languages, Cambridge. Cambridge University Press.

(2004): «Research Perspectives on Teaching English as a Lingua Franca», Annual Review of Applied Linguistics, 24: 209-239.

SifAKIS, N. C. (2004): «Teaching EIL - Teaching International or Intercultural English? What Teachers Should Know», System, 32: 237-250.

SifaKis, N. C.; A. M. Sougari (2005): «Pronunciation Issues and EIL Pedagogy in the Periphery: A Survey of Greek State School Teachers' Beliefs», TESOL Quarterly, 39 (3): 467-488.

TAYLOR, L. (1993): Pronunciation in Action, Hertfordshire, Prentice Hall International (English Language Teaching).

Usó, L. (2007): Creencias de los profesores de E/LE sobre la enseñanza/aprendizaje de la pronunciación, tesis doctoral inédita dirigida por Dr. Francisco José Cantero Serena, publicada por la Universitat de Barcelona. DL: B.48402-2008.

$<$ http:/www.tdx.cat/bitstream/handle/10803/1295/LUV_TESIS.pdf?sequence=1 $>$. 
(2009): «Creencias de los profesores en formación sobre la enseñanza de la pronunciación», MarCoELE, 8: 1-32.

$<$ http://marcoele.com/descargas/uso_creencias.pdf $>$.

Verdía, L. (2010): «Variables afectivas que condicionan el aprendizaje de la pronunciación: reflexión y propuestas», MarcoELE, 10: 223-242. $<\mathrm{http}: / /$ marcoele.com/descargas/expolingua_2002.verdia.pdf $>$.

Williams, M ; R. Burden (1999): Psicología para profesores de idiomas. Enfoque del constructivismo social, Madrid, CuP. 